\title{
Geometrical Optics from the Ground up
}

\author{
Jan Vrbik \\ Department of Mathematics, Brock University, St. Catharines, Ontario, Canada \\ Email: jvrbik@brocku.ca
}

How to cite this paper: Vrbik, J. (2020) Geometrical Optics from the Ground up. Applied Mathematics, 11, 1021-1040. https://doi.org/10.4236/am.2020.1110068

Received: September 15, 2020

Accepted: October 23, 2020

Published: October 26, 2020

Copyright $\odot 2020$ by author(s) and Scientific Research Publishing Inc. This work is licensed under the Creative Commons Attribution International License (CC BY 4.0).

http://creativecommons.org/licenses/by/4.0/

\begin{abstract}
We review, with proper derivation and proofs, the common undergraduate formulas for building images of objects using a system of lenses with spherical surfaces. This is done using the first-order approximation which assumes that light rays deviate from the symmetry axis by only small angles. Yet, even this most basic approximation results in surprisingly complex theory, which is then applied to explain workings of everyday optical instruments.
\end{abstract}

\section{Keywords}

Paraxial Approximation, Cardinal Points, Ray-Transfer Matrix, Keystone Distortion, Exit Pupil, Aparture and Vignetting

\section{Introduction}

Undergraduate Physics curriculum usually includes a brief introduction to Geometrical Optics, but students are often given a few basic formulas to learn only how to apply them, using graphs as the primary tool. The derivation of these formulas is left to more advanced books (such as [1]) whose level of presentation makes them too difficult as supplementary reading for most undergraduates (the corresponding literature is vast and easily searchable; we make no attempts at its review).

The article's goal is to bridge this gap and make the underlying theory more accessible; the only prerequisites are: a basic knowledge of vectors and two by two matrices, and some prior exposure to the topic itself, roughly at the level of [2] (a classic Physics textbook). Following individual steps of our reasoning should give students a better appreciation of the subject and demonstrate how intimately are realities of our world linked to Mathematics. Our presentation may be also of interest to people from other fields (Mathematics in particular), wishing to familiarize themselves with a new and interesting subject, without having to read an extensive monograph. 
Throughout the presentation we assume that light is a collection of rays emanating, as straight lines, from a light source (all physical objects in daylight are such sources); the wavelike nature of light is totally ignored. When reaching a boundary between two optical media with different speeds of light (light travels faster in air than in water or glass), a ray instantaneously changes its direction before continuing in a straight-line path again. This is referred to as the light's refraction; it enables us to trace light rays passing through an optical system consisting of one or more lenses. Light can also be reflected by some surfaces due to space limitations, we do not discuss the related issue of optical mirrors and their applications. For the same reason, we do not include numerical examples and limit the number of graphs (a multitude of these is easily found on the internet; readers are also encouraged to draw their own, as an aid to proper understanding of individual formulas).

We then investigate what happens when a conical pencil of light rays emanating from a point-like source enters an axially symmetric system consisting of several different optical media, separated from each other by spherically shaped boundaries. We then trace a ray's path as it changes direction at each boundary until it emerges from the last surface. We thus discover that all these rays converge, to a good approximation, to a single point, which to our eyes appears to be a nearly perfect image of the original object. To end up with this, rather idealized picture, we need to assume that the rays' directions always deviate from the axis of symmetry by only small angles; we can then replace $\sin \alpha$ of any such angle by $\alpha$ itself (in radians). We also ignore the fact that light consists of different colours; we assume, slightly incorrectly, that colour does not affect the light's speed.

This enables us to show how properties of any such optical system can be summarized by the location of a handful of so-called cardinal points (focal and principal points in particular), derive formulas for finding the image of any specific object, and explain basic principles behind common optical instruments (loupe, camera, projector, microscope, telescope and periscope).

\section{First-Order Approximation}

When a ray of a unit direction $\boldsymbol{v}$ (or, more explicitly $\left\langle v_{x}, v_{y}, v_{z}\right\rangle$; this is the notation we use later on) hits a smooth surface separating one optical medium from the next, the new unit direction becomes

$$
\boldsymbol{u}=\frac{\boldsymbol{v}+\boldsymbol{m}\left(\sqrt{n^{2}-1+(\boldsymbol{v} \cdot \boldsymbol{m})^{2}}-\boldsymbol{v} \cdot \boldsymbol{m}\right)}{n}
$$

where $n$ is the speed of light in the original medium, divided by the speed of light in the new medium, this is called the relative index of refraction, and $\boldsymbol{m}$ is a unit vector, normal to the surface at the point of the ray's entry and oriented into the new medium; this implies that both $\boldsymbol{v} \cdot \boldsymbol{m}$ and $\boldsymbol{u} \cdot \boldsymbol{m}$ are positive. Note that all our directions and normal vectors are of unit length. 
Proof. Basic Physics tells us that $\boldsymbol{u}$ must be in the plane defined by $\boldsymbol{v}$ and $\boldsymbol{m}$ (having the form of $a \boldsymbol{v}+b \boldsymbol{m}$ clearly meets this condition), and it must also comply with Snell's law, which reads

$$
1-(\boldsymbol{v} \cdot \boldsymbol{m})^{2}=n^{2}\left(1-(\boldsymbol{u} \cdot \boldsymbol{m})^{2}\right)
$$

where the dot products $\boldsymbol{v} \cdot \boldsymbol{m}$ and $\boldsymbol{u} \cdot \boldsymbol{m}$ provide the cosines of the corresponding angles. The usual version of the law states that the ratio of the sin's of the two angles is equal to $n$; our formulation is clearly equivalent.

To show that (1) complies with (2), take the dot product of each of its sides with $\boldsymbol{m}$ (recall that $\boldsymbol{m} \cdot \boldsymbol{m}=1$ ) and multiply by $n$, thus getting

$$
n(\boldsymbol{u} \cdot \boldsymbol{m})=\sqrt{n^{2}-1+(\boldsymbol{v} \cdot \boldsymbol{m})^{2}}
$$

Squaring each side of (3) and subtracting from $n^{2}$ yields (2).

Finally, (3) makes $\boldsymbol{u} \cdot \boldsymbol{m}$ positive, implying that $\boldsymbol{u}$ has the correct orientation. Note that $n^{2}-1+(\boldsymbol{v} \cdot \boldsymbol{m})^{2}$ must be positive to achieve refraction, rather than total reflection in this article, we do not need to consider the latter possibility.

When the $x$ and $y$ components of all three ( $u, v$ and $\boldsymbol{m})$ vectors are small, (1) can be, to a linear accuracy discarding second and higher powers of small quantities, simplified to

$$
\left\langle u_{x}, u_{y}\right\rangle=\frac{\left\langle v_{x}, v_{y}\right\rangle+(n-1)\left\langle m_{x}, m_{y}\right\rangle}{n}
$$

since, in this approximation, $\boldsymbol{v} \cdot \boldsymbol{m} \simeq 1$. Note that the $z$ component of all our unit vectors is approximately equal to 1 , thus becoming a redundant piece of information (our notation simply leaves it out).

All subsequent equations are correct only to the linear accuracy; this is referred to as paraxial approximation.

\subsection{Ray Tracing}

Consider a ray that starts at $\langle x, y, 0\rangle$, the so-called object (note that our "objects" are single points, but are often represented by an arrow from $z$ axis to this point), and follows direction $\boldsymbol{v}$, at a small angle to the $z$ axis (horizontal, in our graphs) until it reaches a spherical-surface medium of relative index $n$. Note that the sphere's radius $R$ is measured from the apex of this surface, whose $z$ coordinate is $g$, to the sphere's center, as in Figure $1 ; R$ is thus negative for concave surfaces and infinite for flat surfaces (most of our quantities may have negative values; this does not affect our formulas, they remain correct without a need for any modification).

The point of entry into the new medium is therefore $\langle x, y, 0\rangle+g\left\langle v_{x}, v_{y}, 1\right\rangle$, making the normal direction to the surface at this point equal to

$$
\left\langle\frac{x+g v_{x}}{R}, \frac{y+g v_{y}}{R}\right\rangle
$$




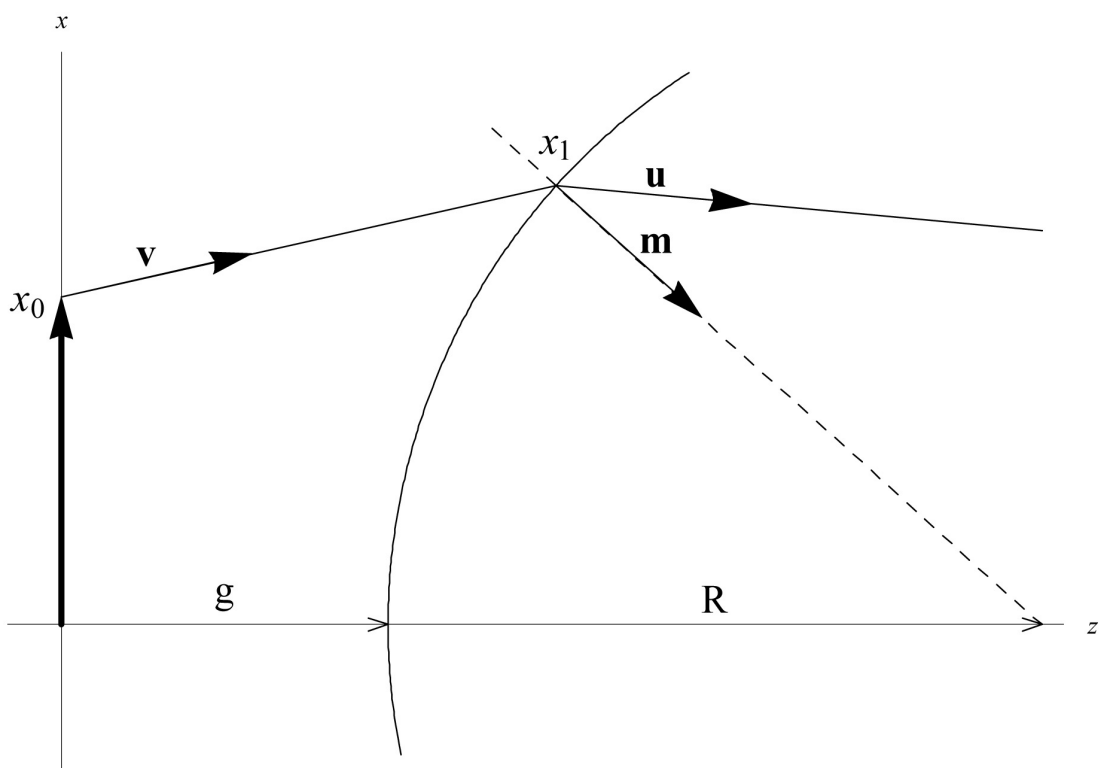

Figure 1. Ray refraction.

Combined with (4), this yields the ray's new direction, namely

$$
\left\langle\frac{v_{x}}{n}+\frac{(n-1)\left(x+g v_{x}\right)}{n R}, \frac{v_{y}}{n}+\frac{(n-1)\left(y+g v_{y}\right)}{n R}\right\rangle
$$

Based on the last expression, we conclude that it is sufficient to follow a ray's path in the $x-z$ plane only, since its $x$ and $y$ components fully decouple (are algebraically independent of each other), implying that results obtained for the $x$ component then have their automatic analog (just replace $x$ by $y$ and $v_{x}$ by $v_{y}$ ) in the $y$ direction. This makes it unnecessary to consider the $y$ coordinate any further; from now on, we discard it and work strictly in the two-dimensional $x-Z$ plane. We can then correspondingly simplify our notation: $v$ will stand for the original $v_{x}$, and our object may now be placed at $\left\langle x_{0}, 0,0\right\rangle$, instead of $\langle x, y, 0\rangle$.

\subsection{Matrix Notation}

Based on these results, the $x$ component of the ray's new location and of its direction can then be conveniently computed using the usual transfer-matrix technique (see [3]); note that location and direction have become elements of a one-column matrix.

$$
\left[\begin{array}{l}
x_{1} \\
v_{1}
\end{array}\right]=\left[\begin{array}{cc}
1 & 0 \\
\frac{1-n_{1}}{n_{1} R_{1}} & \frac{1}{n_{1}}
\end{array}\right]\left[\begin{array}{ll}
1 & g \\
0 & 1
\end{array}\right]\left[\begin{array}{l}
x_{0} \\
v_{0}
\end{array}\right] \stackrel{\text { def }}{=} \mathbb{S}_{1} \mathbb{T}_{0}\left[\begin{array}{l}
x_{0} \\
v_{0}
\end{array}\right]
$$

where subscripts 0 and 1 now indicate the original and the new values respectively; similarly subscripting $R$ and $n$ implies that the ray has just entered the first such surface.

Extending this to a system of $k$ axially symmetrical, spherical surfaces, each with its own $R, n$ (relative to the previous medium) and distance $q_{j}$ from the 
apex of the previous, $f^{\text {th }}$ surface (where $j=1,2, \cdots, k-1$ ), we get the $x$ coordinate and direction of the ray at the point of leaving the last surface by a simple iteration (i.e. repeated application) of (7), namely

$$
\left[\begin{array}{c}
x_{k} \\
v_{k}
\end{array}\right]=\mathbb{S}_{k} \mathbb{T}_{k-1} \cdots \mathbb{S}_{2} \mathbb{T}_{2} \mathbb{S}_{1} \mathbb{T}_{1} \mathbb{S}_{0}\left[\begin{array}{l}
x_{0} \\
v_{0}
\end{array}\right]
$$

where

$$
\mathbb{S}_{j}:=\left[\begin{array}{cc}
1 & 0 \\
\frac{1-n_{j}}{n_{j} R_{j}} & \frac{1}{n_{j}}
\end{array}\right] \text { and } \mathbb{T}_{j}:=\left[\begin{array}{cc}
1 & q_{j} \\
0 & 1
\end{array}\right]
$$

It is convenient to rewrite (8) as

$$
\left[\begin{array}{l}
x_{k} \\
v_{k}
\end{array}\right] \stackrel{\operatorname{def}}{=}\left[\begin{array}{ll}
A & C \\
B & D
\end{array}\right] \mathbb{T}_{0}\left[\begin{array}{l}
x_{0} \\
v_{0}
\end{array}\right]
$$

(slightly departing from the usual notation of other authors). Note that the determinant of $\mathbb{S}_{j}$ (of $\mathbb{T}_{j}$ ) is equal to $\frac{1}{n_{j}}$ (to 1 ); therefore, when the final medium has the same speed of light as the original one (which is the most common situation, as the ambient medium of a system of lenses is usually the air), the product of all these determinants must equal to 1 . This further implies that, in such a case, $\Delta:=A D-C B=1$. Nevertheless, most of our subsequent formulas are not making this assumption, to keep them fully general; note that $\Delta$ must be always positive.

\subsection{Image Creation}

When a ray originating at an object leaves the last surface of such a system and continues for a horizontal distance $h$, its location and direction are then given by

$$
\left[\begin{array}{ll}
1 & h \\
0 & 1
\end{array}\right]\left[\begin{array}{ll}
A & C \\
B & D
\end{array}\right]\left[\begin{array}{ll}
1 & g \\
0 & 1
\end{array}\right]\left[\begin{array}{l}
x_{0} \\
v_{0}
\end{array}\right]=\left[\begin{array}{c}
(A+B h) x_{0}+(C+D h+A g+B h g) v_{0} \\
B x_{0}+(D+B g) v_{0}
\end{array}\right]
$$

Now, consider a pencil (implying: many different $v_{0}$ values) of such rays originating at $\left\langle x_{0}, 0,0\right\rangle$ and converging to a single point again, thus creating an image of the original object where all these rays intersect. This clearly happens at the distance $h_{i}$, obtained by making the coefficient of $v_{0}$ in the expression for the final $x$ location, the first component of (11), equal to 0 , i.e. by solving

$$
C+g A+D h+g B h=0
$$

for $h$. This gives

$$
h_{i}=-\frac{g A+C}{g B+D}
$$

yielding the following $X$ coordinate of the resulting image

$$
\left(A+B h_{i}\right) x_{0}=\frac{A D-C B}{g B+D} x_{0}=\frac{\Delta}{g B+D} x_{0} \stackrel{\text { def }}{=} M x_{0}
$$

where $M$ is the corresponding magnification (often negative, indicating that 
the image is upside down or inverted); when $|M|<1$, the image is smaller than the object, making "magnification" an euphemism. Note that $h_{i}$ may also turn out to be negative; this then implies that the image is only virtual, as the outgoing rays actually diverge (only by extending them backwards makes them converge to an image; our eyes can see it there, but it cannot be captured on film). A real (as opposed to virtual) image can be imprinted onto a light-sensitive layer of "receptors" (to use a generic term), placed at the distance $h_{i}$ from the last surface.

In this context, it is important to mention that rays preserve their colour and intensity (at least proportionately: a brighter object creates a brighter image) of the light of their source. Similarly, one has to realize that an optical system hardly ever deals with a single object, it is always a continuous multitude of them (we call it a "scenery"), transforming them into the corresponding multitude of images. Our formulas imply that images of objects with the same value of $g$ (defining the so-called object plane) form a re-scaled (by factor $M$ ), but otherwise undistorted and sharp image of the original scenery at distance $h_{i}$ from the last surface (in a $z$-perpendicular image plane), often captured on a planar layer of film. Point-like objects at distance $g^{\prime} \neq g$ will appear on this film as small, uniformly illuminated disks, whose radius is proportional to $\left|g^{\prime}-g\right|$; we call such images "out of focus".

\subsection{Focal Points}

From (13), it is easy to see that, when the distance from the object to the first surface is

$$
g=-\frac{D}{B} \stackrel{\text { def }}{=} f_{1}
$$

the image (i.e. the value of $h_{i}$ ) is at infinity, implying that the outgoing rays are parallel to each other; $f_{1}$ thus establishes the $z$ location of the first focal plane, perpendicular to the $z$ axis; this plane intersects the $z$ axis at the first focal point (labelled $f_{1}$ in Figure 2; but otherwise, use definition (15) in all subsequent formulas).

Proof. The fact that the outgoing rays must be parallel follows from

$$
\left(\left[\begin{array}{ll}
A & C \\
B & D
\end{array}\right]-\frac{D}{B}\left[\begin{array}{ll}
0 & A \\
0 & B
\end{array}\right]\right)\left[\begin{array}{l}
x_{0} \\
v_{0}
\end{array}\right]=\left[\begin{array}{c}
A x_{0}+\frac{\Delta}{B} v_{0} \\
B x_{0}
\end{array}\right]
$$

since the final direction is the same for all values of $v_{0}$.

This implies that the final direction of any incoming ray passing through the first focal point (i.e. when $x_{0}=0$ ) is equal to 0 , the ray must come out of the system parallel to $Z$, note that when $f_{1}$ is negative, the incoming ray needs to be extended to have it cross the focal point.

Similarly, as $g$ approaches infinity, implying that the incoming rays from any such distant object become parallel to each other, the corresponding $h_{i}$ tends to 


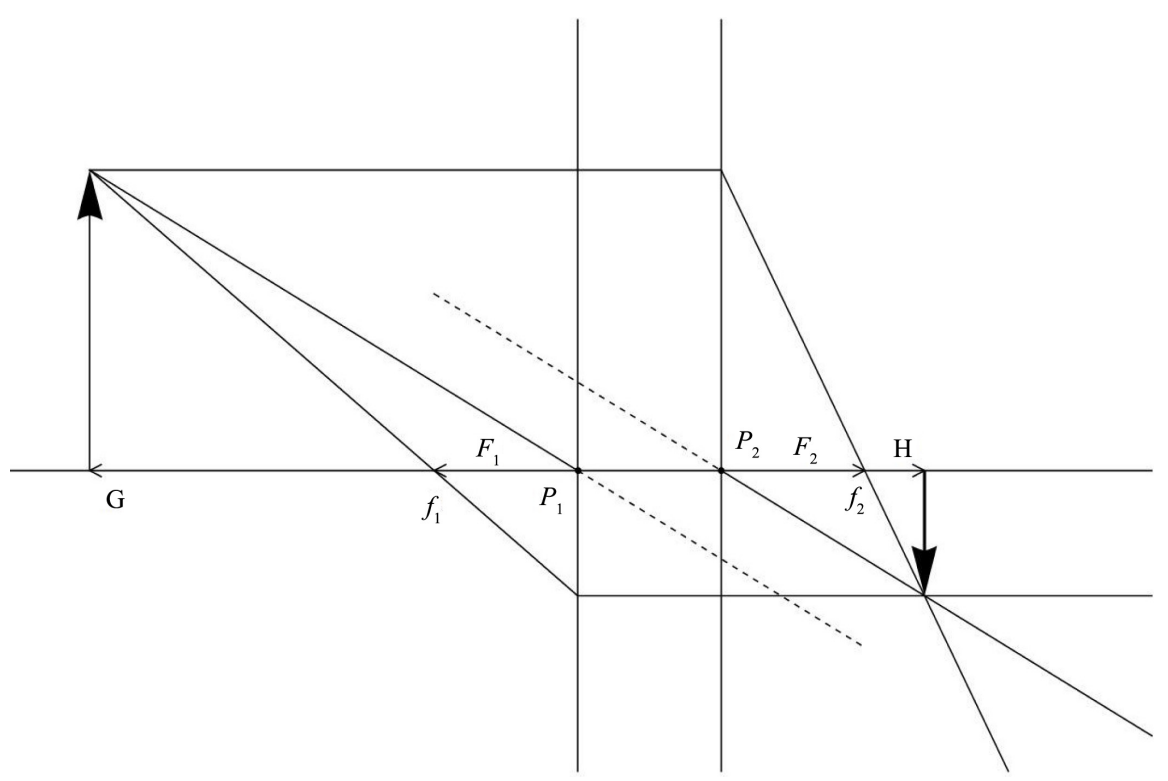

Figure 2. Image construction.

$$
f_{2}:=-\frac{A}{B}
$$

which yields the $z$ distance from the last surface to the second focal plane, where the resulting image is located. In general: incoming parallel rays always converge to a single point in the second focal plane.

Proof. The $x$ coordinate of a ray (which starts with arbitrary values of $x_{0}$ and $v_{0}$ ) at distance $f_{2}$ from the last surface is given by

$$
\left[\begin{array}{ll}
1 & -\frac{A}{B}
\end{array}\right]\left(\left[\begin{array}{ll}
A & C \\
B & D
\end{array}\right]+g\left[\begin{array}{ll}
0 & A \\
0 & B
\end{array}\right]\right)\left[\begin{array}{l}
x_{0} \\
v_{0}
\end{array}\right]=-\frac{\Delta}{B} v_{0}
$$

Rays with the same value of $v_{0}$ (regardless of $x_{0}$ ) must therefore intersect at this same point.

This further implies that a ray originally parallel to $z$ (i.e. having $v_{0}=0$ ) must pass, upon leaving the system, through the second focal point; when $f_{2}$ is negative, this statement becomes "virtual", i.e. the outgoing straight line, when extended backwards, passes through this point.

\subsection{Principal Points}

The value of $g$ which results in unit magnification (i.e. $M=1$ ) is, based on (14), equal to

$$
p_{1}:=\frac{\Delta-D}{B}
$$

This defines the location (in terms of its $z$ distance to the apex of the first surface) of the first principal point and of the corresponding, perpendicular to $z$, principal plane, as most of our distances, $p_{1}$ may turn out to be negative. The image of a (potentially virtual) object whose $g=p_{1}$ is then located in the second principal plane, which crosses the $z$ axis at the second principal point and whose $z$ 
distance from the last surface (again, possibly negative) is, based on (13),

$$
p_{2}:=-\frac{p_{1} A+C}{p_{1} B+D}=-\frac{A \frac{\Delta}{B}-\frac{\Delta}{B}}{\Delta}=\frac{1-A}{B}
$$

The significance of the two principal planes is this: they allow us to simplify the path of a ray entering a system of several surfaces by letting the incoming straight line reach the first principal plane, then continue by a horizontal line to the second principal plane, from where the new path follows the correct final direction, given by the second component of (11). The result is clearly not a true representation of the ray's full path, but it is a proper way of connecting its incoming and outgoing parts and thus sufficient for finding the image of an object, as done in the next section.

Proof. Assuming that the ray starts at $\left\langle x_{0}, 0,0\right\rangle$, all we have to do is to show that the new, simplified path, being given the correct outgoing direction, has also the correct $x$ value, i.e. (14), at distance (13) from the last surface. This is confirmed by

$$
\begin{aligned}
x_{0}+\left(g-p_{1}\right) v_{0}+\left(h_{i}-p_{2}\right) v_{k} \\
=x_{0}+\left(g-\frac{\Delta-D}{B}\right) v_{0}+\left(-\frac{g A+C}{g B+D}-\frac{1-A}{B}\right)\left(B x_{0}+v_{0}(B g+D)\right) \\
=\left(g-\frac{\Delta-D}{B}-g A-C-g(1-A)-\frac{(1-A) D}{B}\right) v_{0} \\
+\left(1-(1-A)-\frac{(g A+C) B}{g B+D}\right) x_{0} \\
=\left(\frac{g B A+D A-g A B-C B}{g B+D}\right) x_{0}=\mathrm{Mx}_{0}
\end{aligned}
$$

Note that it is possible to reverse the logic of the last step of this construction: with the help of principal planes, we can construct the outgoing ray when, instead of knowing its final direction, we know the exact location of the image; after reaching the second principal plane, we run a straight line through the image.

\subsection{Main Formulas}

We now derive a simple formula relating the following two focal lengths, namely

$$
\begin{aligned}
& F_{1}:=f_{1}-p_{1}=-\frac{\Delta}{B} \\
& F_{2}:=f_{2}-p_{2}=-\frac{1}{B}
\end{aligned}
$$

to distances of the object (image) to (from) the first (second) principal plane, namely

$$
\begin{aligned}
& G:=g-p_{1}=g-\frac{\Delta-D}{B} \\
& H:=h_{i}-p_{2}=-\frac{g A+C}{g B+D}+\frac{A}{B}-\frac{1}{B}=\frac{\Delta}{B(g B+D)}-\frac{1}{B}
\end{aligned}
$$


Having defined these, it is easy to show that

$$
\frac{F_{1}}{G}+\frac{F_{2}}{H}=1
$$

Proof.

$$
\frac{\Delta}{\Delta-D-g B}+\frac{1}{1-\frac{\Delta}{g B+D}}=\frac{\Delta-g B-D}{\Delta-D-g B}=1
$$

when $\Delta=1$ (the ambient medium is air), $F_{1}=F_{2}$, with the corresponding simplification of (24). Let us also note that object-related distances such as $G$ and $F_{1}$ have opposite orientation to $H$ and $F_{2}$, as seen in Figure 2.

The RHS of (14) then provides a quick and simple way of finding the image's $x$ location, where magnification is now computed by

$$
\mathrm{M}=\frac{F_{1}}{F_{1}-G}
$$

Proof.

$$
\frac{F_{1}}{F_{1}-G}=\frac{\Delta}{g B-(\Delta-D)+\Delta}=\frac{\Delta}{g B+D}
$$

Similarly, solving (24) for $H$ yields the image's $z$ location, namely

$$
H=\frac{F_{2}}{1-\frac{F_{1}}{G}}=\frac{G F_{2}}{G-F_{1}}
$$

and provides an alternate formula for

$$
\mathrm{M}=\frac{F_{2}-H}{F_{2}}
$$

In addition to this algebraic way of finding the image, the simplified-path construction of the previous section provides an equivalent geometrical technique of achieving the same goal by:

- starting at the object, run a straight line parallel to $z$ axis till reaching the second principal plane, then continue by a straight line, running it through the second focal point,

- run a straight line from the object through the first focal point till reaching the first principal plane, then continue parallel to $z$.

The image is located where these two lines intersect. A simple graph of Figure 2 confirms that this is equivalent to (26) and (28).

This means that, in the current approximation, optical properties of any system of lenses are fully specified by the location of the two focal and the two principal points.

\subsection{Nodal Points}

There is one more pair of the so called cardinal points, defined in the following 
manner.

For each object, it is possible to find a ray whose incoming and outgoing segments are parallel to each other; this happens when the outgoing direction, the second component of (11), is equal to $v_{0}$, implying

$$
v_{0}=\frac{B x_{0}}{1-D-g B} \stackrel{\text { def }}{=} v_{*}
$$

The incoming ray then intersects the $z$ axis at

$$
-\frac{x_{0}}{v_{*}}=\frac{D-1}{B}+g
$$

thus defining the first nodal point, whose distance to the first surface is then $g$ minus (31), namely

$$
d_{1}:=\frac{1-D}{B}
$$

Similarly, the emerging ray intersects the $z$ axis at the second nodal point, whose distance from the last surface is

$$
\begin{aligned}
d_{2} & :=h_{i}-\frac{\mathrm{M} x_{0}}{v_{*}}=-\frac{g A+C}{g B+D}-\frac{\Delta(1-g B-D)}{B(g B+D)} \\
& =-\frac{g A B+A D-\Delta(g B+D)}{B(g B+D)}=-\frac{A-\Delta}{B}
\end{aligned}
$$

The two nodal points enable us to add yet another path to our geometric construction of the image, namely: a straight line from the object to the first nodal point, horizontally connected to the second nodal point, and then continued in the original direction, as shown in Figure 2; note that the graph assumes that $\Delta=1$, resulting in its simplification, as described shortly.

Defining

$$
\begin{aligned}
& F_{1}^{\prime}:=f_{1}-d_{1}=-\frac{1}{B} \\
& F_{2}^{\prime}:=f_{2}-d_{2}=-\frac{\Delta}{B}
\end{aligned}
$$

and

$$
\begin{aligned}
& G^{\prime}:=g-d_{1}=g-\frac{1-D}{B} \\
& H:=h_{i}-d_{2}=-\frac{g A+C}{g B+D}+\frac{A}{B}-\frac{\Delta}{B}=\frac{\Delta}{B(g B+D)}-\frac{\Delta}{B}
\end{aligned}
$$

enables us to derive a twin equation to (24), namely

$$
\frac{F_{1}^{\prime}}{G^{\prime}}+\frac{F_{2}^{\prime}}{H^{\prime}}=1
$$

Proof.

$$
\frac{1}{1-D-g B}+\frac{1}{1-\frac{1}{g B+D}}=\frac{1-g B-D}{1-D-g B}=1
$$


when $\Delta=1$, there is no difference between principal and nodal points, i.e. $d_{1}=p_{1}$ and $d_{2}=p_{2}$; furthermore $F_{1}=F_{2}=F_{1}^{\prime}=F_{2}^{\prime}$, resulting in a welcomed simplification of all previous formulas. This is what is assumed in our next section.

\subsection{Simple Lens}

For a lens with two spherical surfaces $R_{1}$ and $R_{2}$ of apex-to-apex distance $q$, made of a medium (usually glass) with an index of refraction $n$, we get, based on (10)

$$
\begin{aligned}
{\left[\begin{array}{ll}
A & C \\
B & D
\end{array}\right] } & =\left(\left[\begin{array}{cc}
1 & 0 \\
\frac{n-1}{R_{2}} & n
\end{array}\right]+q\left[\begin{array}{cc}
0 & 1 \\
0 & \frac{n-1}{R_{2}}
\end{array}\right]\right)\left[\begin{array}{cc}
1 & 0 \\
\frac{1-n}{n R_{1}} & \frac{1}{n}
\end{array}\right] \\
& =\left[\begin{array}{cc}
1-\frac{(n-1) q}{n R_{1}} & \frac{q}{n} \\
(n-1) \frac{n\left(R_{1}-R_{2}-q\right)+q}{n R_{1} R_{2}} & 1+\frac{(n-1) q}{n R_{2}}
\end{array}\right]
\end{aligned}
$$

Formula (22) then yields the lens' focal length

$$
F=\frac{n}{n-1} \cdot \frac{R_{1} R_{2}}{n\left(R_{2}-R_{1}+q\right)-q}
$$

while, from (32), we get the distance of the first nodal point to the first spherical surface

$$
p_{1}=\frac{R_{1} q}{n\left(R_{2}-R_{1}+q\right)-q}
$$

and, from (33), the distance of the second nodal point from the second spherical surface

$$
p_{2}=\frac{-R_{2} q}{n\left(R_{2}-R_{1}+q\right)-q}
$$

The distance from $p_{1}$ to $p_{2}$ is thus equal to

$$
\Gamma:=\frac{\left(R_{1}-R_{2}\right) q}{n\left(R_{2}-R_{1}+q\right)-q}+q=\frac{q(n-1)\left(R_{2}-R_{1}+q\right)}{n\left(R_{2}-R_{1}+q\right)-q}
$$

Formula (39) is often presented in the following form

$$
\frac{1}{F}=(n-1)\left(\frac{1}{R_{1}}-\frac{1}{R_{2}}+\frac{(n-1) q}{n R_{1} R_{2}}\right)
$$

For a so-called thin lens, $q$ can be approximated by zero, yielding, for the focal length's reciprocal, the following simple expression

$$
(n-1)\left(\frac{1}{R_{1}}-\frac{1}{R_{2}}\right)
$$

while both $p_{1}$ and $p_{2}$ are then equal to zero. 


\subsection{Keystone Distortion}

Another interesting consequence of our main formulas (even though of lesser practical importance) is that objects located in the same object plane (this time not necessarily perpendicular to $z$ ) form their images in the corresponding image plane (see the subsequent proof); when the planes are tilted with respect to $Z$, the collection of such images builds a sharp but distorted rendition of the original scenery, since magnification varies with the image's $z$ coordinate, this creates the so-called keystone effect (a square tilted on one of its sides becomes a trapezoid, the shape of a keystone; a different picture emerges when a square array of dots is tilted on one of its vertices, as Figure 3 demonstrates).

Proof. Going back to the full, three-dimensional description, we consider objects at $\langle x, y, a x+b y+c\rangle$ where $a, b$ and $c$ are fixed parameters, while $x$ and $y$ have variety of values (thus defining an arbitrary plane); note that the first refracting surface is now placed at $z=0$. This implies that the corresponding $G_{x, y}=-a x-b y-c-p_{1}$ and the resulting image is thus at

$$
\mathbf{I}:=\left\langle\frac{F_{1} x}{F_{1}-G_{x, y}}, \frac{F_{1} y}{F_{1}-G_{x, y}}, p_{2}+\frac{G_{x, y} F_{2}}{G_{x, y}-F_{1}}\right\rangle
$$

which is a parametric ( $x$ and $y$ are now the variable "parameters", just a semantic switch) representation of the image plane. The same plane is more conventionally defined by one of its points, namely

$$
\mathbf{I}_{0}:=\left\langle 0,0, p_{2}+\frac{\left(c+p_{1}\right) F_{2}}{c+p_{1}+F_{1}}\right\rangle
$$

found by evaluating $\mathbf{I}$ at $x=y=0$, and the following normal (but not necessarily unit) vector

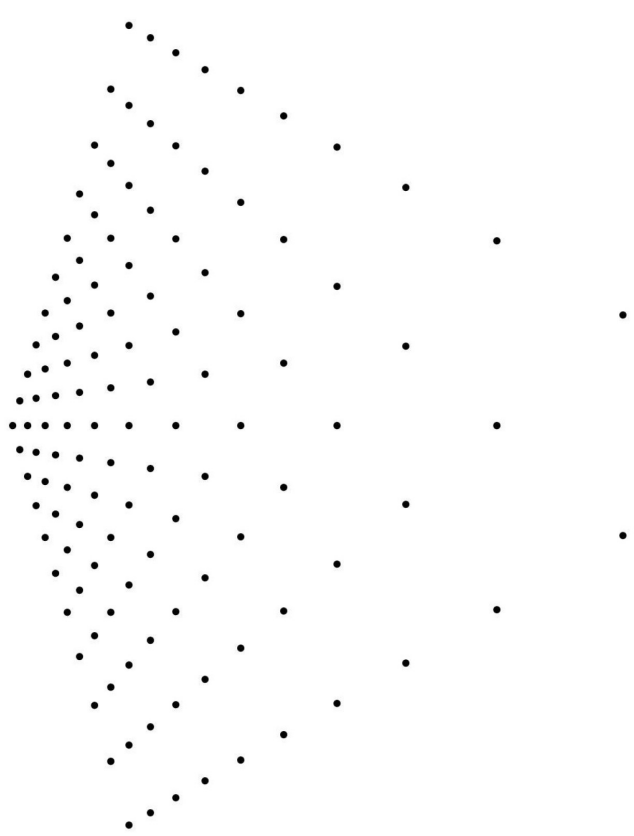

Figure 3. Keystone distortion. 


$$
\mathbf{N}:=\left\langle a F_{2}, b F_{2},-\left(c+p_{1}+F_{1}\right)\right\rangle
$$

This is verified by showing that the dot product of $\mathbf{I}-\mathbf{I}_{0}$ and $\mathbf{N}$ equals to zero for any $x$ and $y$, to simplify this task, we first multiply the former vector by $F_{1}-G_{x, y}$. The corresponding dot product is then

$$
\begin{aligned}
& a x F_{1} F_{2}+b y F_{1} F_{2}-\left(c+p_{1}+F_{1}\right) G_{x, y} F_{2}-\left(c+p_{1}\right) F_{2}\left(F_{1}-G_{x, y}\right) \\
& =(a x+b y) F_{1} F_{2}+F_{1} G_{x, y} F_{2}+\left(c+p_{1}\right) F_{2} F_{1} \\
& =-\left(c+p_{1}\right) F_{1} F_{2}+\left(c+p_{1}\right) F_{2} F_{1}=0
\end{aligned}
$$

Similarly one can show that an image of a straight line is also a straight line.

Proof. Now, the object is at $\langle x, a x+c, b x+d\rangle$ where $x$ is arbitrary, implying that $G_{x}=-b x-d-p_{1}$, and yielding the following parametric representation of the image line and one of its points

$$
\begin{gathered}
\mathbf{I}:=\left\langle\frac{F_{1} x}{F_{1}-G_{x}}, \frac{F_{1}(a x+c)}{F_{1}-G_{x}} y, p_{2}+\frac{G_{x} F_{2}}{G_{x}-F_{1}}\right\rangle \\
\mathbf{I}_{0}:=\left\langle 0, \frac{F_{1} c}{d+p_{1}+F_{1}}, p_{2}+\frac{\left(d+p_{1}\right) F_{2}}{d+p_{1}+F_{1}}\right\rangle
\end{gathered}
$$

respectively. Since $\mathbf{I}-\mathbf{I}_{0}$ is proportional to the constant vector

$$
\left\langle F_{1}+d+p_{1},\left(F_{1}+d+p_{1}\right) a-b c, F_{2} b\right\rangle
$$

for any value of $x$ (just divide the former by $F_{1} x$ and multiply by the common denominator), the line is straight.

The keystone distortion thus presents the original scenery as if seen from a different perspective.

\section{Optical Instruments}

We now turn our attention to more practical issues (see [4]), discussing basic principles behind common optical instruments, including some technical challenges which arise in this context. Since the ambition of this article does not go beyond the first-order approximation, we avoid the topic of optimizing the quality of resulting images by using groups of lenses (more on this in our Conclusion); we aim at a rudimental understanding of workings of these instruments only.

\subsection{Loupe}

When a lens is used as a magnifying glass ("loupe" is an alternate name, usually applied to the watchmaker's version), the object is placed slightly to the right (in terms of our $z$ axis) of the first focal plane ( $F$ must be positive), so as to create its virtual image at $H \simeq-25 \mathrm{~cm}$ (since $25 \mathrm{~cm}$ is the closest distance a normal eye can focus at). From (24) we find that, to achieve this, we need

$$
G=\frac{F}{1-\frac{F}{H}}=\frac{F}{1+\frac{F}{25}}
$$


where $F$ is also in $\mathrm{cm}$. The image thus appears on the object's side of the lens; it is then viewed (from the opposite side) with the observer's eye close to the lens, implying that the corresponding magnification (26) is

$$
\frac{F}{F-G}=\frac{\left(1+\frac{F}{25}\right) F}{\left(1+\frac{F}{25}-1\right) F}=\frac{25}{F}+1
$$

The image is thus bigger (by the extra $\frac{25}{F}$ term) than the actual object as it would appear when viewed from the same $25 \mathrm{~cm}$ distance without a magnifying glass.

A few things to note:

- To achieve an actual, i.e. bigger than 1 , magnification, $F$ must be positive (as mentioned already).

- The image is upright (or erect) rather than inverted, since $M$ is positive.

- When the object is placed directly in the first focal plane, (26) becomes infinite, but consequently not very meaningful; what needs to be compared now is the image's angular size (regular size divided by the image's distance, which is given by $\frac{M x_{0}}{-H}$ and tends to $\frac{x_{0}}{F}$ as $H$ approaches infinity) with the object's angular size when viewed from a distance of $25 \mathrm{~cm}$ (i.e. $\frac{x_{0}}{25}$ ), resulting in $\frac{25}{F}$. The advantage of this arrangement is that the observer's eyes do not need to strain; the image appears at infinity, the most comfortable focus for a normal eye, but the resulting magnification is then smaller than (53).

In this context we should mention that the eye itself is a rather amazing optical instrument, consisting of a lens of adjustable focal length and aperture; with the help of the brain, it is capable of building a three-dimensional (due to having two eyes, which see each object from slightly different angles) and sharp (due to its ability to change its focus and direction so quickly that it can scan the whole scenery in an instant) representation of the outside world.

Some eyes have difficulty focusing over the usual $25 \mathrm{~cm}$ to infinity range; this can by corrected by placing a pair of simple lenses with the appropriate focal length in front of them. We have thus described the most common optical instrument: the eyeglasses.

\subsection{Object (Image) at Infinity}

When $g$ is orders of magnitude larger than the size of the optical system itself (e.g. a camera in a landscape mode, an eye watching a distant object, etc.), rather than converting the existing formulas to their $g \rightarrow \infty$ limit (which would be rather difficult), it is more expedient to

- have a pencil of rays starting at the object reach the first surface as parallel lines, 
- identify the object's location by the value of $v_{0}$ (instead of $x_{0}$, which would be infinite as well),

- use $g=0$; for each ray of the pencil, $x_{0}$ is then the $x$ coordinate of where the ray enters the first surface.

The image is found at the point of convergence of the outgoing rays; this, as we already know, happens at the second focal plane. The corresponding $x$ value (the image's size) is, based on (11) with $g=0$ and $h=f_{2}$, equal to

$$
\left(C-\frac{A}{B} D\right) v_{0}=-\frac{\Delta}{B} v_{0}=F_{1} v_{0}
$$

Since the image needs to be real (as opposed to virtual), $F_{1}$ needs to be positive. As $v_{0}$ is the incoming angle, rays from objects above the $z$ axis arrive with negative values of $v_{0}$ (and vice versa); the camera thus builds an inverted picture of the scenery.

Conversely, when placing an object of vertical size $x_{0}$ in the first focal plane, we know that the outgoing rays of the corresponding pencil are parallel to each other, leaving the system at an angle given by (11) with $g=f_{1}$, namely

$$
B x_{0}=-\frac{x_{0}}{F_{2}}
$$

This describes, to a good approximation, objects (originally captured on a planar film) being projected onto a large distant screen. The image is again inverted; this is easily fixed by inverting the film instead. $F_{2}$ must be positive since the film needs to be located before the first surface and be properly illuminated. To get the image's size, it is sufficient to multiply (55) by the screen's distance.

The illumination is facilitated by a small source of intense light directed at the film. This necessitates placing an extra collector lens between the source and the film, to make the light converge at the projecting lens; also: having a spherical mirror behind the source to reflect its rays in the film's direction.

\subsection{Microscope and Telescope}

The same approach can be also used to explain workings of a microscope: a tiny object of size $x_{0}$ is similarly "projected" to create a real image at a relatively large distance $t$ (called tube length); the resulting magnification is then given by the size of this image (see the previous section) divided by $x_{0}$, i.e.

$$
-\frac{x_{0}}{F_{2}} \times t \div x_{0}=-\frac{t}{F_{2}}
$$

The lens, called an objective, must therefore have a small focal length to achieve high magnification. The image thus created is then observed through (becomes the object of) another, ocular lens, also called an eyepiece, which functions in a manner of a loupe; typically, an ocular is a system of two or more lenses, to improve its optical properties. The overall magnification of the microscope is then the product of (56) and (53).

Finally, a telescope (a pair of these makes so called binoculars) is an arrange- 
ment of two lenses such that the first focal point of the second (ocular) lens coincides with the second focal point of the first (objective) lens. The object is effectively at infinity, which means that its location must be specified by the angle, say $v_{0}$, at which parallel rays from the object enter the first lens. Based on (54), this creates an image of size $\dot{F}_{1} v_{0}$ (a single dot implying the first lens) in the objective's second focal plane. The ocular (again, essentially a loupe) then converts this image into a pencil of parallel rays leaving the telescope at an angle given by (55), namely

$$
-\frac{\dot{F}_{1}}{\ddot{F}_{2}} v_{0}
$$

(two dots implying the second lens). The coefficient of $v_{0}$ is the corresponding angular magnification (to make it large, $\dot{F}_{1}$ must be close to the tube length, whereas $\ddot{F}_{2}$ is kept relatively small, several ocular lenses may be interchangeably used with the same telescope, to allow for different overall magnifications).

When both focal lengths are positive, the image is inverted, when $\dot{F}_{1}$ is positive (as it must be, to create a real image) and $\ddot{F}_{2}$ is negative, the image is upright; the latter design is used only for relatively small magnifications (opera glasses, etc.). Using the former design for land-based binoculars (where an erect image is essential inversion makes no difference to astronomers) necessitates inserting, between the objective and the ocular, either four reflecting mirrors (this is what makes the usual binoculars so bulky), or an extra erector lens. This lens takes the image created by the objective, making it its object, and creates a new image in the ocular's first focal plane, using 1 magnification (achieved by $G=2 F$ and $H=2 F$, where $F$ is the erector' $s$ focal length). This increases the length of the instrument by $4 F+\Gamma$, where $\Gamma$ was defined in (42); such a design is used mainly in rifles' telescopic sights, which need to be of a small diameter at the expense of a longer tube.

We mention in passing that wave properties of light put a limit on magnification achievable in this manner.

\subsection{Combining Two Systems}

Assuming that the ambient medium is air and, consequently, $\Delta=1$, we now explore what happens when two such optical systems (e.g. two surfaces of a simple lens; a tandem of two lenses; objective and ocular of an instrument; etc.) are combined by arranging them, one after the other, along the $z$ axis. Assuming that the principal points and the focal length of each of the two systems are known (we place single and double dot over quantities of the first and second system, respectively) and taking the distance from $\dot{p}_{2}$ to $\ddot{p}_{1}$ to be $s$ (potentially negative), we want to find $p_{1}, p_{2}$ and $F$ of the new, combined system, as these fully determine its overall optical properties; the two individual sub-systems no longer matter. We claim that

$$
p_{1}=\dot{p}_{1}-\frac{\dot{F} S}{\dot{F}+\ddot{F}-S}
$$




$$
\begin{gathered}
p_{2}=\ddot{p}_{2}+\frac{\ddot{F} s}{\dot{F}+\ddot{F}-s} \\
F=\frac{\dot{F} \ddot{F}}{\dot{F}+\ddot{F}-s}
\end{gathered}
$$

where $p_{1}\left(p_{2}\right)$ is the distance of the first (second) principal point of the combined system to (from) the first (last) surface of the first (second) sub-system.

Proof. Placing on object at $p_{1}$ which, from the perspective of the first sub-system, see (23), corresponds to

$$
\dot{G}=p_{1}-\dot{p}_{1}=-\frac{\dot{F} s}{\dot{F}+\ddot{F}-s}
$$

we get, based on (28), for the location of the resulting image (specifically, its distance from $\dot{p}_{2}$ )

$$
\dot{H}=\frac{\dot{G} \dot{F}}{\dot{G}-\dot{F}}=\frac{-\dot{F}^{2} s}{-\dot{F} s-\dot{F}^{2}-\dot{F} \ddot{F}+\dot{F} s}=\frac{\dot{F} s}{\dot{F}+\ddot{F}}
$$

implying that

$$
\ddot{G}=s-\dot{H}=\frac{\ddot{F} s}{\dot{F}+\ddot{F}}
$$

The combined system's magnification is clearly just a product of the two individual magnifications, namely

$$
\frac{\dot{F}}{\dot{F}-\dot{G}} \cdot \frac{\ddot{F}}{\ddot{F}-\ddot{G}}=\frac{\dot{F}^{2}+\dot{F} \ddot{F}-\dot{F} s}{\dot{F}^{2}+\dot{F} \ddot{F}} \cdot \frac{\dot{F} \ddot{F}+\ddot{F}^{2}}{\dot{F} \ddot{F}+\ddot{F}^{2}-\ddot{F} s}=1
$$

resulting in overall unit magnification. This proves that $p_{1}$ is the correct first principal point of the combined system.

From the perspective of the second sub-system, an object at $\ddot{G}$, given by (63), has its image at the following distance from $\ddot{p}_{2}$

$$
\ddot{H}=\frac{\ddot{G} \ddot{F}}{\ddot{G}-\ddot{F}}=\frac{\ddot{F}^{2} s}{\ddot{F} s-\dot{F} \ddot{F}-\ddot{F}^{2}}=\frac{\ddot{F} s}{s-\dot{F}-\ddot{F}}
$$

which verifies (59).

Finally, starting with an object at $\dot{G}=\infty$ yields $\dot{H}=\dot{F}, \ddot{G}=s-\dot{F}$ and

$$
\ddot{H}=\frac{\ddot{G} \ddot{F}}{\ddot{G}-\ddot{F}}=\frac{\ddot{F} s-\dot{F} \ddot{F}}{s-\dot{F}-\ddot{F}}
$$

Converting $\ddot{H}$ to the distance from $p_{2}$ (instead of $\ddot{p}_{2}$ ) yields

$$
F=\frac{\ddot{F} s-\dot{F} \ddot{F}}{s-\dot{F}-\ddot{F}}+\ddot{p}_{2}-p_{2}=\frac{\ddot{F} s-\dot{F} \ddot{F}}{s-\dot{F}-\ddot{F}}+\frac{\ddot{F} s}{\dot{F}+\ddot{F}-s}=\frac{\dot{F} \ddot{F}}{\dot{F}+\ddot{F}-s}
$$

In the same manner, it is possible to combine three or more such optical sub-systems into a single one, whose principal points and focal length (and again: to the current level of approximation, nothing else is needed to know the combined system's optical properties) are then established by a repeated application of (58) to (60). 
Formula (60) explains workings of the so-called zoom lens: by changing the value of $s$, we can give the combined system an almost arbitrary $F$, i.e. the corresponding angle-to-size magnification given by (54). Note that the smallest possible value of $s$ is $-\left(\dot{p}_{2}+\ddot{p}_{1}\right)$ when the physical distance between the two original sub-systems becomes zero, they come into direct contact with each other.

We need to mention that the actual design of a camera's objective (think $\mathrm{Ni}$ kon, Zeiss, Canon, etc.) involves many additional considerations and requires more sophisticated mathematical formulation to achieve a sharp and undistorted image for a continuous variety of magnifications.

\subsection{Aperture and Vignetting}

Our discussion would not be complete without considering the following issue: in addition to the geometric properties discussed up to this point, it is also important for an optical system to let through the maximum amount of light, to make the corresponding image as bright as possible. To achieve this goal, we must first have some way of establishing which rays of a pencil starting at an object actually make it to their target, i.e. to the corresponding image.

To determine that, we realize that all spherical surfaces of an optical system have a finite radial extent; we can then visualize them as a slightly curved disks of different diameters. Note that the curvature is now irrelevant; it is their circular contour which matters.

By tracing individual rays of the original pencil, we discover that not all of them can reach the image, as some simply miss one of these disks and get absorbed by the instrument's enclosure. To find exactly which rays do succeed, we create images of all these disks as seen from the object side, i.e. by reversing the rays' direction (the first disk is an image of itself, the second one has its image created by the first surface, etc.); the smallest one of the resulting disk images (called the entrance pupil) corresponds to the so-called aperture, i.e. the actual, physical surface behind this image. A ray makes it through the whole system if and only if it can pass through the aperture; equivalently, an incoming ray (extended, if necessary) must pass through the entrance pupil, and a (potentially extended) outgoing ray must pass through the corresponding exit pupil (the aperture's image when viewed from the image side of the system). Looking through binoculars from a distance against a white background, the disk of light we see is the exit pupil; by slightly moving the binoculars we may notice that this pupil is located in front of the ocular; the corresponding distance is called eye relief. Ideally, the location of the exit pupil should be where the eye is normally placed, and its diameter should match the eye's pupil (about $7 \mathrm{~mm}$ ). Similarly, looking through binoculars from a distance, but this time from the wrong side, the white disk we see is the entrance pupil, i.e. another image of the same aperture.

Since the entrance images of the previous paragraph are all disks centered on the $z$ axis, they appear concentric to an object near the $z$ axis; we already know 
that only by passing through the smallest one of these (the entrance pupil) will allow a ray pass through the whole system. When the object is off the $z$ axis, the disks' centers are no longer concentric but appear aligned along a straight line; they move further apart as the object's distance from the $z$ axis increases. For a ray to pass through the whole system now requires it to pass through all these disks, i.e. through what appears (from the object's point of view) their overlap. The area of this overlap decreases as the object moves further away from the $Z$ axis (this effect is called vignetting), until the edge of one of the bigger disks (an image of a physical surface called the field stop) reaches the center of the entrance pupil; the angular location of such an object defines the system's field of view. Move the object a bit further and it becomes practically invisible to the observer.

An optical system (such as a camera) may have a deliberate additional light barrier (called a diaphragm) with adjustable circular opening to function as the system's aperture; this allows us to control the film's exposure. Similarly, the field of view is usually restricted by the physical extent of the film itself, which then defines the system's field stop.

\subsection{Periscope}

When one part of a system (usually, the objective) creates an intermediate image which is then taken to be the object of the systems's next part (the ocular), as done in telescopes, microscopes, etc., it is possible to improve the system's light-transmitting properties by inserting, at the intermediate image/object location, an extra (so-called field) lens with positive $F$. Its function is to move the image/object from its first to its second principal plane, leaving it otherwise intact (i.e. using $M=1$ ), while bending the passing rays so as to prevent them from hitting the instrument's tube.

This idea is essential for building systems which are limited in diameter but whose tube needs to be fairly long (such as periscopes). It should be intuitively obvious that even after precisely aligning the objective and ocular parts of such a long and narrow system (by itself a daunting task, especially when the tube may need to bend, as in endoscopes), the amount of light reaching the ocular would be extremely small (if any). To fix this problem, we need to go back to the idea of an erector lens (which takes an image and makes its inverted carbon copy at the distance $4 F+\Gamma$ ), and insert as many of these as needed to link the objective with the ocular; this alleviates the problem of alignment. At the same time, halfway between these relay (as they are now known) lenses, it is necessary to insert the same number of field lenses described in the previous paragraph, to ensure that enough light gets through; each of these lenses adds only $\Gamma$ to the instrument's length.

In endoscopes, it is necessary that the relay/field lenses have even smaller axial diameter, but are rather long (surface to surface); they are then called rod lenses. Arranging them next to each other (in spite of their different functions, they can be otherwise identical) then results in leaving only small air gaps between them, 
thus allowing the tube to be both stiff (in terms of its length) and flexible (in terms of its bending). To ensure proper illumination, sufficient light needs to be transmitted through an endoscope, starting from its image end (where the eye is); after brightening the object, the reflected light then travels back to the observer.

\section{Conclusions}

In this article we have described only the most basic properties of a system of lenses; the attentive reader may have realized that, if everything was as perfect as presented here, there would be no need for using more than one lens for any given purpose (only its cardinal points and focal length would matter). This is definitely not the case in real world, and it is now time to admit that the actual situation is somehow more complicated. This is due to so-called aberrations (lens properties causing image distortion and smearing, problems with proper focusing, etc.), which arise when third and higher-order terms are included in the expansion of the sin function. The second important source of these is the fact that most optical materials (glass in particular) have an index of refraction which slightly varies with the light's colour.

To minimize these imperfections (in order of their seriousness and this would vary from one type of optical instrument to another), instead of single lens, one must use a complex system of several lenses with different indices of refraction, designed in a way to make their individual aberrations cancel each other. This is most important in the design of camera objectives, which must achieve such cancellation for objects at many different distances (not to mention a further complication of zooming, i.e. changing the resulting magnification). No wonder that these often require a collection of twelve or more lenses, not all of them with spherical surfaces (this provides the designer with extra flexibility). Such aberrations will be discussed in proper detail in our subsequent submission.

\section{Conflicts of Interest}

The author declares no conflicts of interest regarding the publication of this paper.

\section{References}

[1] Herzberger, M. (1980) Modern Geometrical Optics. Krieger Pub. Co., Malabar.

[2] Halliday, D., Resnick, R. and Walker, J. (2013) Chapter 34, Fundamentals of Physics. Wiley, Hoboken.

[3] Lin, P.D. (2016) Advanced Geometrical Optics. Springer, Berlin. https://doi.org/10.1007/978-981-10-2299-9

[4] Smith, W.J. (2008) Modern Optical Engineering. $4^{\text {th }}$ Edition, McGraw-Hill, location 2 Pennsylvania Plaza New York City. 